\title{
Flora terrestre de la isla Malpelo (Colombia), Pacífico Oriental Tropical
}

\author{
Rubén D. González-Román ${ }^{1}$, Mateo López-Victoria ${ }^{2}$ \& Philip A. Silverstone-Sopkin ${ }^{1}$ \\ 1. Grupo de Investigación en Florística; Departamento de Biología, Facultad de Ciencias Naturales y Exactas, Calle 13 \\ \# 100-00, Universidad del Valle, Cali, Colombia; ruben.gonzalez@correounivalle.edu.co, \\ pasilverstone_sopkin@yahoo.com \\ 2. Departamento de Ciencias Naturales y Matemáticas, Facultad de Ingeniería, Calle 18 No. 118-250, Pontificia \\ Universidad Javeriana-Cali, Cali, Colombia; malov@javerianacali.edu.co
}

Recibido 05-IV-2013. Corregido 09-IX-2013. Aceptado 09-X-2013.

\begin{abstract}
Terrestrial flora of Malpelo Island, Colombia, Eastern Tropical Pacific. Malpelo Island is located $380 \mathrm{~km}$ off the mainland continental coast of Colombia, in the Pacific Ocean. Several geological, ecological, and zoological studies, both marine and terrestrial, have been conducted in this island. Despite some marginal comments on some publications, no single specific survey has been devoted to botany so far. In order to make a floristic inventory of the terrestrial flora of this island, three field trips were made in 2010 to collect vascular plants, mosses, and lichens, as well as data on their distribution within the island. We collected and identified 25 species of lichens, two species of vascular plants and one moss. Lichens were the most diverse group found, including records of four new genera (Endocarpon, Fuscidea, Lecanographa and Verrucaria) and 13 new species for Colombia. The high lichen richness on Malpelo might be explained by their efficient form of asexual reproduction (soredia and isidia), that may have facilitated their transport to the island by migrating birds or wind. Once on the island, it is possible that lichens persist by being chemically protected against herbivores. The great number of new generic and species records for Colombia is explained by the low number of studies in saxicolous lichens conducted so far in the country, particularly on coastal areas and remote islands. Only two species of vascular plants were collected, a grass, Paspalum sp., and a fern, Pityrogramma calomelanos, and both of them correspond to new determinations for Malpelo. A moss species previously reported but with no positive identification was collected and identified as Octoblepharum albidum. Other species previously reported, for example, some species of shrubs, were not observed. The low number of vascular plants is probably due to a combination of soil conditions and herbivory by land crabs. This study is the first complete inventory of the flora of Malpelo and is a starting and reference point for future comparisons among islands in the Eastern Tropical Pacific. Rev. Biol. Trop. 62 (1): 327-336. Epub 2014 March 01.
\end{abstract}

Key words: Colombia, Malpelo Island, vascular plants, Bryophyte, saxicolous lichens.

Aunque a primera vista Malpelo parece inhabitable, como un monolito basáltico que emerge del mar hasta alcanzar $300 \mathrm{~m}$ de altura, se destacan la existencia y las adaptaciones propias para sobrevivir en el sustrato rocoso de unas cuantas especies de flora y fauna terrestres, algunas de ellas endémicas: el cangrejo de Malpelo Johngarthia malpilensis Faxon, los lagartos Anolis agassizi Stejneger, Phyllodactylus transversalis Huey y Diploglossus millepunctatus O'Shaughnessy, y los caracoles Malpelina labiata e Ischnocion conica
Hausdorf et al. También hacen parte de la fauna de la Isla más de 80 invertebrados, pertenecientes a varios órdenes (Calero, López-Victoria \& Chacón de Ulloa, 2011), y la mayor colonia reproductiva de aves marinas en Colombia, compuesta principalmente por el Piquero de Nazca, Sula granti Rothschild, con una población adulta estimada en 80000 individuos (López-Victoria \& Rozo, 2007).

Procesos de erosión y meteorización, la oferta de guano, las lluvias y la condensación del vapor de agua han permitido el 
establecimiento de algunos organismos autótrofos en Malpelo: algas, líquenes, musgos, gramíneas, leguminosas y helechos (Wolda, 1975). Sin embargo, debido en apariencia al nivel de aislamiento geográfico de Malpelo, y a la pobre disponibilidad y calidad de los suelos y nutrientes, no se ha desarrollado una comunidad vegetal muy diversa (Murphy, 1945; Wolda, 1975; López-Victoria, 2009). Otra causa propuesta como explicación de la ausencia de vegetación superior es la presencia del cangrejo terrestre Johngarthia malpilensis, cuya población se estima en casi un millón de individuos, ya que se trata de un organismo omnívoro que se alimenta de casi cualquier cosa, incluyendo retoños de plantas y ropa (López-Victoria \& Werding, 2008; López-Victoria, 2009).

Debido a la ausencia de productores primarios de importancia en Malpelo, la mayoría de los animales terrestres de la Isla depende directa o indirectamente de la energía proveniente del océano, en especial la aportada por el Piquero de Nazca en forma de excrementos, polluelos, huevos y plumas (López-Victoria, Wolters \& Werding, 2009). La escasa cubierta vegetal presente recibe el guano de las aves (que actúa como abono) y es consumida mayoritariamente por lagartos, cangrejos y, en menor proporción, por otros invertebrados terrestres (como hormigas, grillos, caracoles, miriápodos e isópodos), siendo estos herbívoros y omnívoros los únicos eslabones entre los organismos autótrofos y el resto del ecosistema de la Isla (López-Victoria \& Werding, 2008; López-Victoria, 2009; López-Victoria et al., 2009).

Solo Wolda (1975) hizo algunas observaciones generales sobre la flora de Malpelo: escribió sobre una especie de gramínea ( $\sin$ identificar), un helecho (Pityrogramma dealbata), una probable leguminosa y sobre la facilidad para encontrar líquenes (Caloplaca sp., Candelaria sp., Aspicilia sp., Diplochistes sp., Lecidea sp., Basidea sp., Pyxine cf. glebosa y una Graphidaceae sin identificar) y musgos. Posteriormente, López-Victoria y Rozo (2006) calcularon la cobertura vegetal en Malpelo y en los islotes aledaños, principalmente del helecho; también encontraron un pasto y algunos arbustos no identificados y sin recolectar, y registraron la presencia permanente de microalgas, líquenes y musgos (también sin identificar). Mientras que la flora de Malpelo se ha mantenido casi inexplorada, muchos trabajos de inventarios florísticos se han llevado a cabo en islas de la región. Vale la pena mencionar los estudios realizados en la isla Gorgona (Colombia; Murillo \& Lozano, 1989), en las islas Galápagos (Ecuador; Wiggins \& Porter, 1971), en la isla Barro Colorado (Panamá; Croat, 1978), en la isla del Coco (Costa Rica; Trusty, Kesler \& Delgado, 2006) y en las islas Marietas (México; Ramírez-Delgadillo et al., 2000), por mencionar algunos. En el caso de todas esas islas, las comunidades vegetales terrestres están compuestas principalmente por plantas vasculares.

Debido al aislamiento geográfico y a las condiciones extremas para trabajar en Malpelo, su flora no ha sido estudiada en profundidad, y existen muy pocas observaciones publicadas sobre este tema (Rand, Gorman \& Rand, 1975; Wolda, 1975; López-Victoria \& Rozo, 2006). El objetivo de este estudio fue realizar el primer inventario detallado y sistemático de la flora terrestre de la isla Malpelo, incluyendo musgos, helechos, angiospermas, líquenes y hongos no liquenizados. El presente estudio constituye un punto de referencia y comparación para estudios semejantes en otras islas del Pacífico Oriental Tropical.

\section{MATERIALES Y MÉTODOS}

Área de estudio: Malpelo es una isla oceánica, localizada en el Pacífico colombiano

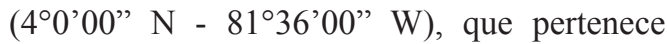
al municipio de Buenaventura (departamento del Valle del Cauca) y se encuentra a $380 \mathrm{~km}$ del punto más cercano de la costa continental colombiana. Presenta un área emergida de $1.2 \mathrm{~km}^{2}$, una altura máxima de $300 \mathrm{~m}$ y está rodeada de 11 islotes (López-Victoria \& Rozo, 2006). Se trata de una isla oceánica, de origen volcánico, que es la cima de una cordillera submarina denominada Dorsal de Malpelo (Stead, 1975). La isla principal tiene de largo $1643 \mathrm{~m}$, 
y su ancho máximo es $727 \mathrm{~m}$. Cerca del $72 \%$ de la superficie de la Isla presenta pendientes muy marcadas; sin embargo, hay algunas áreas planas en los sectores sur, norte y oriental (López-Victoria \& Rozo, 2006). A pesar de su apariencia enteramente rocosa, algunos sectores presentan cantidades considerables de suelo. Aunque no hay datos meteorológicos precisos para Malpelo, se ha estimado preliminarmente una precipitación promedio anual de $2500 \mathrm{~mm}$, y una temperatura promedio de $25^{\circ} \mathrm{C}$ (López-Victoria, datos sin publicar).

Muestreo y determinación: Se hicieron tres visitas a Malpelo durante los meses de febrero, julio y diciembre 2010, con una duración aproximada de seis días de trabajo cada una. Con el fin de realizar el inventario de las especies de organismos autótrofos, se recolectó la mayor cantidad de material posible, incluyendo plantas vasculares, líquenes y musgos. Debido al terreno accidentado, las recolectas de material terrestre se llevaron a cabo en recorridos libres entre los cero y los 300 metros de altura por los sectores occidental y sur de la Isla, así como por los cerros central y sur, y por el sector del Tangón, sin seguir un recorrido de muestreo específico. Se recolectó únicamente material en condiciones ideales para su identificación.

Las plantas vasculares se recolectaron con todas sus partes, incluyendo su raíz o rizoma, y se utilizó una prensa botánica y bolsas plásticas con alcohol al 70\% para su preservación. Los líquenes y musgos se recolectaron con un cincel y un martillo, tomando siempre una muestra que contuviese la totalidad del espécimen (o una muestra lo suficientemente grande para su identificación) y parte del sustrato en el que se encontraba. Los líquenes fueron almacenados en bolsas de papel con sílica gel (para extraer la humedad). Los musgos se recolectaron de forma semejante a los líquenes, pero fueron preservados secos o en alcohol al 70\%. Adicionalmente, se tomaron datos de crecimiento, características que se pueden perder con los métodos de preservación (color y tamaño), altura a la que se encontraba el espécimen y la posición satelital exacta en donde fue recolectado (Trusty et al., 2006).

El material recolectado fue identificado con la ayuda de especímenes de referencia del Herbario CUVC - "Luis Sigifredo Espinal-Tascón" de la Universidad del Valle, empleando diferentes claves dicotómicas para cada grupo, y con el apoyo adicional de algunos expertos en cada grupo (para el caso particular de líquenes se siguió a Sipman, 2006). Posteriormente, todos los especímenes identificados e incluidos en esta publicación fueron depositados en la colección del Herbario CUVC.

Algunos datos de cobertura de líquenes fueron estimados en forma visual, directamente en campo, o a partir de fotografías. Estas estimaciones se presentan como indicativos generales de cobertura, sin llegar a ser mediciones exactas, y tienen un propósito descriptivo.

\section{RESULTADOS}

Un total de 122 muestras de material fueron recolectadas en las tres salidas de campo. El listado de las identificaciones se presenta en el cuadro 1. Se identificaron 19 géneros y 25 especies de líquenes, 16 de las cuales fueron identificadas al nivel de especie, pertenecientes a 12 familias, todas de la división Ascomycota. Trece especies (Agonimia papillata, Amandinea submontana, Arthonia catenatula, Caloplaca cupulifera, Dirinaria consimilis, Endocarpon pallidulum, E. pallidum, Graphis dimidiata, Hyperphyscia minor, Lecanora bruneri, Lecanographa lyncea, Opegrapha aperiens y Porina guentheri) y cuatro géneros (Endocarpon, Fuscidea, Lecanographa y Verrucaria) corresponden a nuevos registros para Colombia, según los catálogos presentados por Aguirre-C (2008a) y Sipman, Hekking y Aguirre-C. (2008). Al menos otras dos especies corresponden a nuevos registros, pero las dos morfoespecies del género Verrucaria encontradas no pudieron ser identificadas a nivel de especie. El sustrato donde se encontraban todas las especies de líquenes recolectadas era roca. 
CUADRO 1

Lista de familias y morfoespecies de organismos autótrofos terrestres de la isla Malpelo

TABLE 1

List of families and morpho-species of terrestrial photosynthetic organisms of Malpelo Island

\begin{tabular}{|c|c|}
\hline Familia & Especie \\
\hline \multicolumn{2}{|c|}{ Plantas no vasculares } \\
\hline Leucobryaceae & Octoblepharum albidum Hedw. López-Victoria, M. \#30; CUVC 048339 \\
\hline \multicolumn{2}{|c|}{ Plantas vasculares } \\
\hline Pteridaceae & Pityrogramma calomelanos (L.) Link. López-Victoria, M. \#19; CUVC 048336 \\
\hline Poaceae & Paspalum sp. López-Victoria, M. \#44; CUVC 048350 \\
\hline \multicolumn{2}{|l|}{ Líquenes } \\
\hline \multirow[t]{2}{*}{ Arthoniaceae } & Arthonia catenulata Ach. López-Victoria, M. \#37; CUVC 048344 \\
\hline & Herpothallon minimum Aptroot \& Lücking. González-Román, R. \#78; CUVC 048358 \\
\hline \multirow[t]{2}{*}{ Caliciaceae } & Amandinea submontana Marbach. González-Román, R. \#69; CUVC 048352 \\
\hline & Baculifera cinerocincta (Müll. Arg.) Marbach. López-Victoria, M. \#1; CUVC 048324 \\
\hline Graphidaceae & Graphis dimidiata Vain. López-Victoria, M. \#4; CUVC 048326 \\
\hline Fuscideaceae & Fuscidea sp. González-Román, R. \#86; CUVC 048366 \\
\hline \multirow[t]{2}{*}{ Lecanoraceae } & Lecanora bruneri Imshaug \& Brodo. López-Victoria, M. \#3; CUVC 048325 \\
\hline & Lecanora sp. González-Román, R. \#70; CUVC 048353 \\
\hline Lecideaceae & Lecidea sp. González-Román, R. \#72; CUVC 048354 \\
\hline Parmeliaceae & Parmotrema sp. González-Román, R. \#79; CUVC 048359 \\
\hline \multirow[t]{2}{*}{ Physciaceae } & Dirinaria consimilis (Stirt.) Awasthi. González-Román, R. \#73; CUVC 048355 \\
\hline & Hyperphyscia minor (Fée) Kalb. González-Román, R. \#82; CUVC 048361 \\
\hline Porinaceae & Porina guentheri (Flotow) Zahlbr. González-Román, R. \#87; CUVC 48365 \\
\hline Ramalinaceae & Ramalina complanata (Sw.) Ach. López-Victoria, M. \#15; CUVC 048333 \\
\hline \multirow[t]{4}{*}{ Roccellaceae } & Lecanographa lyncea (Sm.) Egea \& Torrente. González-Román, R. \#117; CUVC 048384 \\
\hline & Opegrapha aperiens Vain. González-Román, R. \#121; CUVC 048387 \\
\hline & Opegrapha sp.1. González-Román, R. \#91; CUVC 048369 \\
\hline & Opegrapha sp. 2. González-Román, R. \#111; CUVC 048381 \\
\hline \multirow[t]{2}{*}{ Teloschistaceae } & Caloplaca cupilifera (Vain.) Zahlbr. López-Victoria, M. \#36; CUVC 048343 \\
\hline & Flavoplaca citrina (Hoffm.) Th. Fr. López-Victoria, M. \#28; CUVC 048338 \\
\hline \multirow[t]{5}{*}{ Verrucariaceae } & Agonimia papillata (O.E. Erikss.) Diederich \& Aptroot. González-Román, R. \#103; CUVC 048378 \\
\hline & Endocarpon pallidum Ach. González-Román, R. \#95; CUVC 048372 \\
\hline & Endocarpon pallidulum (Nyl.) Nyl. González-Román, R. \#80; CUVC 048360 \\
\hline & Verrucaria sp. 1. González-Román, R. \#127; CUVC 048391 \\
\hline & Verrucaria sp. 2. González-Román, R. \#84; CUVC 048363 \\
\hline \multicolumn{2}{|c|}{ Hongos basidiomicetes no liquenizados } \\
\hline Agaricaceae & Leucocoprinus birnbaumii (Corda) Singer. López-Victoria, M. \#45; CUVC 048323 \\
\hline
\end{tabular}

Con respecto a la distribución de los líquenes en Malpelo, se observó una clara dominancia de las especies del grupo Buellia (sensu lato): Amandinea submontana y Bacullifera cinereocincta se encontraron en toda la Isla, incluyendo las partes planas a levemente inclinadas, junto con las especies de los géneros Flavoplaca, Porina y Lecanora. Sin embargo, en las partes más planas de Malpelo, la cobertura de líquenes fue muy pobre (cerca del $10 \%$ de la superficie). En zonas un poco más inclinadas (cerca de las bases de los cerros), se encontraron comúnmente a Flavoplaca citrina y Verrucaria spp. Las especies Ramalina complanata y Herpothallon minimum se encontraron únicamente en las zonas de pendiente muy inclinada, como en las paredes de los cerros sur y centro, y Parmotrema sp. en la base del cerro 
sur; se observaron las tres especies juntas en las paredes del cerro sur, cerca al sector donde están los helechos rastreros.

Solo se registraron tres especies de plantas en Malpelo: un musgo, un helecho y una gramínea. La posible leguminosa y otros arbustos reportados anteriormente no fueron encontrados en los sitios visitados. El musgo, identificado como Octoblepharum albidum, se encontró creciendo únicamente en las grietas del cerro central, a partir de $100 \mathrm{~m}$ de altura. El helecho, anteriormente identificado como Pityrogramma dealbata (Wolda, 1975), fue determinado como P. calomelanos. Este helecho solo se encontró en el sector sur-oriental de Malpelo, cerca a la base del cerro sur, en un pequeño parche de menos de $0.02 \mathrm{~km}^{2}$ (Fig. 1-A). La gramínea se confirmó como una
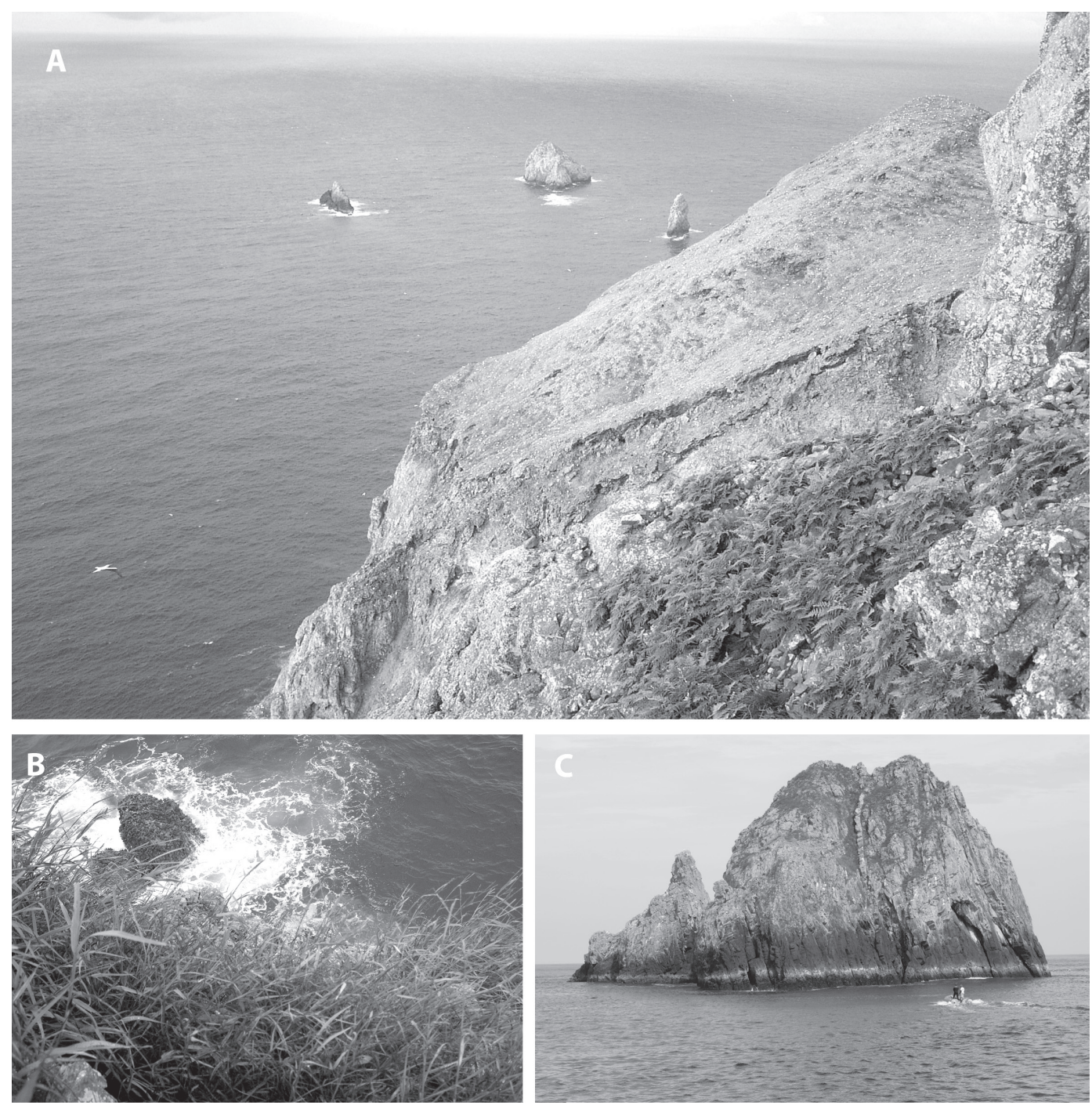

Fig. 1. Parches de vegetación en Malpelo: (A) Pityrogramma calomelanos en el sector sur-oriental. (B) Paspalum sp. en una pared vertical del costado occidental. (C) Paspalum sp. en el islote La Gringa.

Fig. 1. Patches of vegetation on Malpelo Island: (A) Pityrogramma calomelanos in the South-Eastern sector. (B) Paspalum sp. on a vertical wall of the Western side. (C) Paspalum sp. on the islet La Gringa. 
especie del género Paspalum, probablemente dentro del grupo Setacea (Chase), debido a las inflorescencias axilares; a la fecha no ha podido ser identificada a nivel de especie, debido a que su descripción no coincide con la de ninguna otra especie del género (G. Davidse, com. pers.). Este pasto se encontró restringido a unos cuantos islotes del norte y del sur, y a paredes verticales, en la parte alta de los cerros de la isla principal (Fig. 1-B y 1-C).

\section{DISCUSIÓN}

Wolda (1975) realizó el único trabajo donde se mencionan géneros o especies de líquenes para Malpelo, resaltando la presencia de Caloplaca (conocida actualmente como Flavoplaca), Candelaria, Aspicilia, Diplochistes, Lecidea, Basidea, Pyxine y un morfotipo de la familia Graphidaceae, sin identificar las especies. Dos de estos géneros quedan confirmados, y se identificó la especie de la familia Graphidaceae. Sin embargo, los otros géneros que Wolda registró no fueron recolectados durante el presente estudio, porque: a) puede que se haya tratado de errores en las identificaciones presentadas por Wolda, b) esos líquenes no fueron detectados por los investigadores durante las tres salidas de campo del presente estudio, o c) esos géneros se extinguieron en algún momento, entre la visita de Wolda (a comienzos de 1972) y la actualidad (2010).

En ecosistemas insulares marinos y costeros es común encontrar líquenes adaptados a las condiciones particulares de esos ambientes (salinidad, altas temperaturas, resequedad), como son algunas especies de los géneros Verrucaria, Flavoplaca, Ramalina, Arthonia, Dirinaria, Lecanora y Buellia (sensu lato), algunos de ellos formando grandes manchas de colores característicos (amarillo, naranja, rojo, blanco; Rowe \& Egea, 1986; Alonso \& Egea, 1994; Etayo, 1998; Purvis, 2000; Herrera, Aragón, Prieto \& Belinchón, 2007; Rosabal \& Aragón, 2010). Sin embargo, Purvis (2000) señala que, en zonas costeras e islas más cercanas al Ecuador, la combinación del calor extremo y la salinidad disminuye la probabilidad de encontrar las grandes manchas coloreadas o líquenes en la zona intermareal o de salpicadura. Típicamente los líquenes se encuentran a alturas superiores a los $100 \mathrm{~m}$, patrón que parece repetirse en Malpelo, donde la gran mayoría de las muestras se recolectaron en alturas superiores a $100 \mathrm{~m}$, aunque la distribución de algunas especies llegue hasta el nivel del mar. En todo caso, en Malpelo da la impresión de que los líquenes son más frondosos, y sus estructuras reproductivas están en mejor estado, a mayor altura (obs. pers.).

Malpelo nunca ha estado conectada a masas continentales, por lo que se desconoce con exactitud cómo se estableció la comunidad autótrofa en sus superficies. Los líquenes poseen formas de propagación sexuales conocidas como esporas, y hay una creencia general de que estas esporas son de alta duración y amplio espectro de dispersión. Sin embargo, este mecanismo es más complejo, puesto que, aunque la espora pueda llegar a un lugar lejano desde su punto de origen, no todas pueden sobrevivir durante largo tiempo y, en caso de que una espora colonizadora produzca una hifa inicial, necesita encontrar inmediatamente al alga que le servirá como fotobionte, porque de otra manera muere (Purvis, 2000; R. Lücking, com. pers.). El mecanismo más eficiente de dispersión en líquenes es el de reproducción vegetativa por medio de soredios e isidios, ya que éstos contienen tanto al hongo como al fotobionte. En islas remotas, la mayor proporción de líquenes presenta por lo menos una de las dos formas vegetativas de reproducción, y pueden haber llegado allí en las patas de aves migratorias (Purvis, 2000). Puesto que la mayoría de especies de aves en Malpelo son en algún grado migratorias (López-Victoria \& Estela, 2007), esta parece ser la forma en la que llegó la mayoría de especies de líquenes a la Isla; Malpelo es considerada un punto de paso para algunas especies de aves migratorias en sus rutas oceánicas entre Centroamérica y Suramérica (López-Victoria \& Estela, 2007; López-Victoria, 2009).

Los líquenes, que resultan poco comestibles para la mayoría de animales, son 
consumidos ocasionalmente por caracoles, babosas, psocópteros y ácaros, mientras que muy pocos vertebrados consumen líquenes (Purvis, 2000). Salvo los cangrejos terrestres, no hay registros en Malpelo de otros organismos que se alimenten de los líquenes (ni siquiera de las especies de talos foliosos Parmotrema sp. y $R$. complanata). Es probable que las sustancias químicas presentes en los líquenes (metabolitos secundarios) les confieran un sabor amargo o algún tipo de defensa que los hace poco apetecibles para la mayoría de animales (Purvis, 2000), lo que explica su abundancia y riqueza en Malpelo, que contrasta con la escasez notable de plantas vasculares.

El briófito encontrado en Malpelo (Octoblepharum albidum), un musgo de amplia distribución, es cosmopolita, y en Colombia se ha registrado prácticamente en todos los departamentos con alturas que oscilan entre 50 y $1500 \mathrm{~m}$ (Aguirre-C, 2008b). Este musgo también ha sido registrado en los dos sistemas insulares cercanos a Malpelo, isla del Coco y las Galápagos (Williams, 1924).

La especie de helecho registrada en Malpelo figura en la literatura publicada como Pityrogramma dealbata (Wolda, 1975; Prahl, 1990; López-Victoria \& Rozo, 2006). Sin embargo, al correr la clave del género para Guatemala (Stolze, 1981), se ha llegado a la conclusión de que se trata de la especie Pityrogramma calomelanos, debido a la forma de sus pínulas (con el ápice obovado u ovoide en $P$. dealbata y lanceolado en $P$. calomelanos). Trusty et al. (2006) registraron la especie $P$. calomelanos para la isla del Coco, mientras que Wiggins y Porter (1971) reportan P. calomelanos, en su variedad calomelanos, para las islas Galápagos. Pityrogramma dealbata no se encuentra registrada ni en la isla del Coco ni en Galápagos. No obstante, las dos especies son muy similares y pertenecen a un grupo relativamente poco estudiado, que presenta muchas dudas taxonómicas (Knoll \& Machnicki, 2006). Stolze (1981) inclusive sugiere que $P$. dealbata podría ser una subespecie de $P$. calomelanos. Es probable que el helecho esté sufriendo una extinción local en Malpelo, ya que actualmente sólo existe una pequeña porción de la superficie que ocupaba hace 25 a 30 años (López-Victoria \& Rozo, 2006). Las causas de esta posible extinción no están claras, puesto que, aunque el cangrejo $J$. malpilensis se alimenta del helecho, durante al menos tres décadas ambas especies han coexistido en Malpelo (López-Victoria, 2009).

La única angiosperma registrada en Malpelo es el pasto Paspalum sp., que a la fecha no ha podido ser identificada a nivel de especie. Este pasto se encuentra restringido a la cima de unos cuantos islotes y a paredes verticales de la isla principal, en sitios a los cuales el cangrejo terrestre no puede acceder, lo que sugiere que este cangrejo es el moderador de la distribución de esta especie localmente. El género Paspalum es de distribución cosmopolita, y varias especies se encuentran registradas en los dos sistemas insulares oceánicos cercanos a Malpelo: $P$. decumbens, $P$. conjugatum, $P$. virgatum y $P$. nutans para isla de Coco (Trusty et al., 2006) y $P$. conjugatum, $P$. distichum, $P$. vaginatum, $P$. penicillatum, $P$. paniculatum, $P$. redundans, $P$. galapageium para Galápagos (Wiggins \& Porter, 1971); Jaramillo-Díaz, Guézou, Mauchamp \& Tye (2011) listan dos especies más para Galápagos: P. fasciculatum y P. racemosum [los listados de Wiggins \& Porter (1971) y Trusty et al. (2006) incluyen claves dicotómicas para el género Paspalum]. Sin embargo, ninguna de las especies allí registradas concuerda con la especie de Malpelo. En el pasado se realizaron observaciones en Malpelo de otras especies de angiospermas arbustivas (no identificadas ni recolectadas). Aunque en las tres salidas de campo no se observaron otras angiospermas diferentes a Paspalum sp., no puede descartarse del todo su presencia en Malpelo, debido a que varios sectores e islotes aledaños no fueron estudiados en su totalidad, por resultar inaccesibles.

La única especie de hongo no liquenizado registrada en Malpelo corresponde a unas fotografías tomadas en mayo del 2006 y pertenecen a dos individuos de Leucocoprinus birnbaumii, basidiomicete de amplia distribución mundial, muy común en ecosistemas tropicales húmedos y cuyas esporas son fácilmente transportadas 
por el viento (López \& García, 2002; G. Guzmán, com. pers.). Los especímenes de Malpelo se encontraban creciendo en una viga de madera en uno de los baños de la cabaña de la Armada. Aunque su desarrollo fue rápido, poco tiempo después fueron devorados por cangrejos terrestres (López-Victoria, 2009).

$\mathrm{Ni}$ el aislamiento geográfico ni la pobre disponibilidad de suelos parecen justificar la baja riqueza de plantas vasculares y de briófitos, como concluye Wolda (1975). Islas igualmente "aisladas" geográficamente, como la isla del Coco, poseen una comunidad vegetal mucho más compleja, hasta con 263 especies de plantas vasculares registradas (Trusty el al., 2006). Probablemente, algunas de esas especies se establecieron allí gracias a las rutas migratorias de las aves. López-Victoria (2009) demostró que la poca cantidad de suelo que hay en la Isla dispone de suficientes nutrientes para la germinación de semillas y el crecimiento de las plantas. El agua tampoco es un factor limitante para el posible establecimiento de una comunidad vegetal más compleja. Aunque no hay datos precisos sobre precipitación, se estima en al menos $2500 \mathrm{~mm}$ anuales (M. LópezVictoria, datos sin publicar), y hay un constante ingreso de humedad al sistema, como resultado del efecto Massenerhebung: el vapor de agua se condensa en forma de niebla cerca a los cerros y luego, por escorrentía, desciende al resto de la Isla. Es altamente probable que la baja diversidad vegetal (plantas vasculares y briófitos) se deba a factores ecológicos, como la presencia del cangrejo terrestre, combinados con la poca disponibilidad de suelos y sus características (López-Victoria \& Werding, 2008; López-Victoria, 2009). En términos de la relevancia de los resultados del presente estudio, las determinaciones y los nuevos registros para Colombia constituyen el aporte que faltaba a la designación de Malpelo como Santuario de Fauna y Flora del Sistema Nacional de Áreas Protegidas (de Colombia), toda vez que su fauna, pero no su flora, habían sido estudiados y considerados en detalle. Para la región del Pacífico Oriental Tropical, estos registros sirven como punto de referencia para otras islas en la región, y para estudios comparativos futuros.

\section{AGRADECIMIENTOS}

Nuestros agradecimientos a las entidades que hicieron posible este trabajo, tanto financiera como logísticamente: el Instituto de Investigaciones Marinas y Costeras - INVEMAR, la Universidad Justus-Liebig de Giessen (Alemania), la Universidad del Valle, la Pontificia Universidad Javeriana-Cali, la Armada Nacional de Colombia y la Fundación Malpelo y Otros Ecosistemas Marinos. También agradecemos a Enrique Peña-Salamanca (Universidad del Valle) por su acompañamiento y sus importantes aportes durante este proyecto; a Bibiana Moncada (Universidad Distrital Francisco José de Caldas) y a Robert Lücking (The Field Museum) por su colaboración con la identificación de los líquenes; a Gastón Guzmán (Instituto de Ecología, A.C. - México) por confirmar la especie del hongo; a Michael Sundue (New York Botanical Garden) por su ayuda en la determinación del helecho; a Gerrit Davidse (Missouri Botanical Garden) por su ayuda en la determinación del pasto; a Getsy Bolaños (Universidad del Valle) por la colaboración en la identificación del musgo y, especialmente, a Edier Soto-Medina (Universidad del Valle) por su ayuda en la identificación de los líquenes, del hongo y sus aportes en el desarrollo de este proyecto.

\section{RESUMEN}

La isla Malpelo se encuentra en el Pacífico colombiano, a unos $380 \mathrm{~km}$ de la costa continental. La Isla ha sido objeto de algunos estudios geológicos, ecológicos y faunísticos, tanto marinos como terrestres, pero ningún estudio estrictamente botánico. Con el fin de realizar un inventario de la flora terrestre de Malpelo, se realizaron tres salidas de campo durante el 2010, en las que se recolectaron plantas vasculares, musgos y líquenes, y se tomaron datos sobre su distribución en la Isla. El grupo más diverso correspondió a los líquenes, con 25 especies, incluidos cuatro nuevos géneros para Colombia, Endocarpon, Fuscidea, Lecanographa y Verrucaria, y 15 nuevos registros de especies para Colombia. La alta riqueza de especies de líquenes en la Isla puede deberse a la forma de reproducción asexual 
de estos organismos, que pudieron haber sido transportados hasta Malpelo por aves migratorias o por viento; además, pueden estar protegidos químicamente de la herbivoría por cangrejos terrestres. La gran cantidad de registros nuevos para Colombia se explica por la poca cantidad de trabajos en líquenes saxícolas en el país, sobre todo en zonas costeras e insulares. Solo se registraron dos especies de plantas vasculares, una gramínea, Paspalum sp., y un helecho, Pityrogramma calomelanos. También se recolectó una especie de musgo, Octoblepharum albidum. La escasez de plantas vasculares probablemente se deba a la combinación de condiciones de los suelos y a la herbivoría por cangrejos terrestres. El presente estudio es el primero en darle relevancia a la flora terrestre de Malpelo y constituye un punto de referencia y comparación para estudios semejantes en otras islas del Pacífico Oriental Tropical.

Palabras clave: Colombia, isla Malpelo, plantas vasculares, briofitas, líquenes saxícolas.

\section{REFERENCIAS}

Aguirre-C., J. (2008a). Catálogo de los líquenes de Colombia. In: J. O. Rangel (Ed.), Colombia. Diversidad biótica VI. Riqueza y diversidad de los musgos y líquenes en Colombia (pp. 401-547). Bogotá, Colombia: Universidad Nacional de Colombia.

Aguirre-C., J. (2008b). Catálogo de los musgos de Colombia. In: J. O. Rangel (Ed.), Colombia. Diversidad biótica VI. Riqueza y diversidad de los musgos y liquenes en Colombia (pp. 99-319). Bogotá, Colombia: Universidad Nacional de Colombia.

Alonso, F. L., \& Egea, J. M. (1994). Líquenes calcícolas y terrícolas de algunas localidades costeras de Marruecos. Acta Botanica Malacitana, 19, 51-61.

Calero, D., López-Victoria, M., \& Chacón De Ulloa, P. (2011). Composición y estructura trófica de los macroinvertebrados terrestres de la isla Malpelo, Pacífico colombiano. Boletín de Investigaciones Marinas y Costeras, 40, 155-173.

Croat, T. B. (1978). Flora of Barro Colorado Island. Stanford, California, Estados Unidos: Stanford University Press.

Etayo, J. 1998. Aportación a la flora liquénica de las Islas Canarias. iv. Líquenes epífitos de la Gomera (Islas Canarias). Tropical Bryology, 14, 85-107.

Herrera, M., Aragón, G., Prieto, M., \& Belinchón, R. (2007). Diversidad liquénica de las islas Chafarinas, España. Botánica Complutensis, 31, 5-11.

Jaramillo-Díaz, P., Guézou, A., Mauchamp, A., \& Tye, A. (2011). CDF Checklist of Galapagos Flowering Plants. In F. Bungartz, H. Herrera, P. Jaramillo, N. Tirado, G. Jímenez-Uzcategui, D. Ruiz, A. Guézou \& Ziemmeck, F. (Eds.). Charles Darwin Foundation Galapagos Species Checklist. Charles Darwin
Foundation / Fundación Charles Darwin, Puerto Ayora, Galapagos.

Knoll, B. M. J., \& Machnicki, N. J. (2006). Morphological phylogenetic analysis of Costa Rican Pityrogramma (Pteridaceae). Organization for Tropical Studies OTS, Tropical Plant Systematics course, 124-131.

López, A., \& García, J. (2002). Leucocoprinus birnbaumii. Funga Veracruzana 69.

López-Victoria, M., \& Rozo, D. M. (2006). Model-based geomorphology of Malpelo Island and spatial distribution of breeding seabirds. Boletin de Investigaciones Marinas y Costeras, 35, 111-131.

López-Victoria, M., \& Estela, F. A. (2007). Una lista anotada de las aves de la Isla Malpelo. Ornitología Colombiana, 5, 40-53.

López-Victoria, M., \& Rozo, D. M. (2007). Wie viele Nazcatöpel Sula granti brüten auf der Insel Malpelo? Vogelwarte, 45, 365-366.

López-Victoria, M., \& Werding, B. (2008). Ecology of the endemic land crab Johngarthia malpilensis (Decapoda: Brachyura: Gecarcinidae), a poorly known species from the tropical Eastern Pacific. Pacific Science, 62, 483-493.

López-Victoria, M. (2009). Island ecology: trophic and breeding behaviour of land crabs and seabirds determined the terrestrial community of Malpelo. (Tesis de doctorado, Justus-Liebig-Universität, Giessen, Alemania).

López-Victoria, M., Wolters, V., \& Werding, B. (2009). Nazca Booby (Sula granti) inputs maintain the terrestrial food web of Malpelo Island. Journal of Ornithology, 150, 865-870.

Murillo, M. T., \& Lozano, G. 1989. Hacia la realización de una flórula del Parque Nacional Natural Islas de Gorgona y Gorgonilla. Revista de la Academia Colombiana de Ciencias Exactas, Físicas y Naturales, 17, 276-304.

Murphy, R. 1945. Island contrasts. Natural History, 15, 14-23.

Prahl, H. von. (1990). Malpelo la roca viviente. Bogotá, Colombia: Fondo FEN.

Purvis, O. W. (2000). Lichens. Londres, Inglaterra: Natural History Museum.

Ramírez-Delgadillo, R., Cupul-Magaña, F., HernándezHurtado, H., Fonseca-Madrigal, J., Rodríguez-Zaragoza, F., \& Gómez-Graciano, S. (2000). Florística de las islas Marietas, municipio de Bahía de Banderas, Nayarit. Ciencia y Mar, 4, 23-28.

Rand, S. A., Gorman, G. C., \& Rand, W. M. (1975). Natural history, behavior, and ecology of Anolis agassizi. Smithsonian Contributions to Zoology, 176, 27-38.

Rosabal, D., \& Aragón, G. (2010). Líquenes epífitos en el matorral costero de la Reserva Ecológica 
Siboney-Juticí (Cuba). Botanica Complutensis, 34, 21-25.

Rowe, J. G., \& Egea, J. M. (1986). Líquenes silicícolas de las sierras costeras occidentales de la comarca de Algeciras (Cádiz, España). Acta Botánica Malacitana, 11, 55-74.

Sipman, H. J. M. 2006. Lichen determination keys available on internet. Berlin: Botanischer Garten Und Botanisches Museum Berlin-DahleM.

Sipman, H. J. M., Hekking, W., \& Aguirre-C., J. (2008). Checklist of lichenized and lichenicolous fungi from Colombia. Bibliotheca José Jerónimo Triana, 20, 1-242.

Stead, J. A. (1975). Field observations on the geology of Malpelo Island. Smithsonian Contribution to Zoology, 176, 17-20.
Stolze, R. G. (1981). Ferns and fern allies of Guatemala. Part II - Polypodiaceae. Chicago: Fieldiana Botany, new series 6.

Trusty, J. L., Kesler, H. C., \& Delgado, G. H. (2006). Vascular flora of Isla del Coco, Costa Rica. Proceedings of the California Academy of Sciences, 57, 247-355.

Wiggins, I. L., \& Porter, D. M. (1971). Flora of the Galápagos Islands. Stanford: Stanford University Press.

Williams, R. S. 1924. Galapagos and Cocos Island mosses collected by Alban Stewart in 1905-6. The Bryologist, 27, 37-45.

Wolda, H. 1975. The ecosystem on Malpelo Island. Smithsonian Contributions to Zoology, 176, 21-26. 